\title{
Compilation, Validation and Evaluation of a 2D/3D Molecular Database as an Adjunct to Didactic Teaching Modalities
}

\author{
Sultana G., Shoemake C., Camilleri L. \\ University of Malta, Malta
}

\begin{abstract}
Medicinal chemistry is considered an essential component of educational preparation for the pharmacy profession. Effective explanation and comprehension of chemical concepts requires considerable effort due to the intrinsically abstract nature of key principles. The inclusion of two and three dimensional visual adjuncts to traditional lecturing methods provides an innovative and student-inclusive approach towards optimisation of chemistry related education. A molecular database was created that featured therapeutic, physicochemical and visual information of drugs used in infection, musculoskeletal and endocrine disorders. Visuals created include two and three dimensional graphical representations of all the drugs included as well as three dimensional graphics of the drug interacting with its cognate receptor where possible. Expert panel validated pre- and post- intervention questionnaires were then used to evaluate the impact and utility of the two and three dimensional molecular database of therapeutically relevant drugs amongst undergraduate pharmaceutical science students at the Department of Pharmacy of the University of Malta.

The intervention lecture incorporated both traditional teaching and use of the visual adjuncts using case based approaches. The results of paired samples t-tests, the Friedman test and the MannWhitney statistical tests show statistically significant improvement $(p<0.05$ level of significance in all cases) in both student performance and student mentality towards the subject.
\end{abstract}

\section{Introduction}

Medicinal chemistry is a scientific discipline which combines principles from applied sciences, particularly in the field of medicine, and basic sciences where established chemistry conventions are fundamental. Medicinal chemistry is considered a vital facet towards undergraduate training in the pharmaceutical field. It is considered the point of intersection between synthetic chemistry and molecular pharmacology that emphasises the structure-activity relationships (SAR) of drug molecules. Through a clear understanding of the subject matter, pharmacy students acquire the basic knowledge required to derive conclusions about a drug's activity within a biological context from molecular structure. This information can then be extrapolated to the understanding of drugs ${ }^{\text {ee }}$ mechanisms of action, and principles relating to absorption, distribution, metabolism, elimination and toxicity of the drug (ADMETox profile) all of which are important aspects in drug design endeavours [1].

Students who understand these concepts acquire commensurate proficiency in rationally arriving at safe decisions regarding drug selection in clinical and often critical scenarios. Chemical expertise in the mode of action and in vivo disposition of therapeutic molecules singles out pharmacists from other health care professionals. Medicinal chemistry is therefore one of the modules which characterises pharmacists and instills the skills required for competence in the discipline [1].

Chemistry as an area of study is frequently associated with low student performance and enthusiasm. The majority of students follow chemistry courses at undergraduate level as part of obligate credits leading to a qualification in their future professional field. Chemistry is based on abstract concepts which require concentration, time and determination to comprehend; this augments the difficulty associated with the subject. The subject matter should be presented in a visually engaging manner and the main role of educators is therefore to transmit the relevance of undergraduate chemistry modules while clearly portraying abstract concepts by venturing into different teaching methods [2].

\section{Background}

The provision of visual aids to students in order to enhance appreciation of abstract information and improve education outcomes is not an entirely novel concept. Inclusion of visuals offers several advantages to both students and educators. Wileman highlights the following advantages; visuals offer transmission of a larger amount of information over written explanations in the same amount of space, simplification of complicated theories, clarification of abstract, language based concepts, offer organisation of information in the though formation process and most importantly research suggests that 
visuals increase retention of learned material [3]. Studies show that students improve their perception and understanding of the molecular recognition process and may predict molecular properties by handling computer graphics and databases [4]. A comparison of traditional visualisation methods of education such as two dimensional drawings and physical „ball and stick ${ }^{e e}$ models to contemporary computational adjuncts concluded that computer visualisaton of molecules is an effective mode of concept delivery with regards to highly pharmaceutically relevant concepts such as stereochemistry [5].

Two and three dimensional methods of visualisation should be considered complimentary to one another. Two-dimensional images are usually associated with clear, rapid identification of key structural features. Two-dimensional graphics are recently being employed in drug discovery as they allow pharmacologists to rapidly identify and analyse molecular structure. Three-dimensional graphics are associated with an increased fidelity to reality as a depiction but are often considered more difficult to interpret and more time consuming to create and perceive. Depiction of therapeutic molecules in this dimension can therefore give a clear representation of binding pocket-molecule interactions that occur within a biological context such as the human body [6]. Including both types of representations is recommended. The simultaneous presence of two and three dimensional visuals facilitates orientation around the 3D molecule in order to identify the steric factors, isomerism and other spatially conscious concepts [7].

Visual aids within the classroom scenario are not considered beneficial as an exclusive method of presentation of the conceptual material; they provide optimum results when used as supplements to traditional lectures. The implementation of visual models as adjuncts to didactic teaching modalities promotes student participation while providing supplemental information and interactive demonstration of theories. Overall the learning experience was shown to be enhanced during the lecture and the information transmitted showed higher understanding and retention [8]. Traditional didactic, verbal and written methods feature vital knowledge the students must retain, however visual aids and well designed presentation delivery systems enhance student perception and consolidation of material [9].

The combination of linguistic and visual representations provide immediate consolidation of the new concept which promotes knowledge acquisition. This aspect implies that such adjuncts help the memory formation process and enhance learning and understanding. Furthermore, difficulties that may arise in understanding concepts using the didactic approach are resolved by the additional support provided by the visual method. Visual adjuncts also aid in mental categorisation of concepts. This is associated with ease of application in problem-solving scenarios [10]. The caveat lies in the fact that students must be able to interpret the visual graphics into relevant information. Lecturers must be proficient in appropriately highlighting important aspects shown via graphical adjuncts in order to clearly deliver the objective. This introduces the notion of "visual literacy". Wileman defines visual literacy as "the ability to ,read", interpret, and understand information presented in pictorial or graphic images". Closely related to visual literacy is the concept of visual thinking, described as "the ability to turn information of all types into pictures, graphics, or forms that help communicate the information" [3]. These skills imply that there should be a certain basic knowledge amongst both students and lecturers with respect to the presentation and interpretation of graphical information [11]. The use of computerized case studies applied to medicinal chemistry yielded positive results among students who showed improvement in solving relevant therapeutic problems, proper conduction and evaluation of structure-activity relationships and answering patient-centred problems correctly. The structure based therapeutic evaluation (SBTE) approach provides an instructional model providing guidelines for educators to retain basic medicinal chemistry principles while highlighting and applying clinical relevance as part of the curriculum. Innovatively at the time for medicinal chemistry syllabi, it also included summaries of common clinical decisions applied to the drug class and predictions of clinical activity. This approach was received well by students who successfully applied medicinal chemistry concepts to relevant clinical outcomes [12-14].

\section{Methodology}

\subsection{Compilation of Database}

Drugs included in the molecular database were selected using the infection, endocrine and musculoskeletal disorder chapters in version 72 of the British National Formulary (BNF) [15] as a reference. Peptide drugs, ions and combination therapies were excluded due to difficulties in 3D image generation. Datasheets for each chapter were created using a spread sheet. Each drug had its own data entry created within the datasheet featuring the drug name, indications and therapeutic class, all adapted from the drug monographs in the BNF. This data allows correlation of the drugs studied to their use in clinical practice. Highlighting clinical relevance in medicinal chemistry education was seen to have a positive impact in previous studies [12-14] and was therefore incorporated into the database. 
Two-dimensional structures and physicochemical properties such as polar surface area (PSA), partition co-efficient $(\mathrm{A} \log \mathrm{P})$, stereo centre count, hydrogen bond donor and acceptor count, relative molecular mass (RMM), exact mass, chemical formula and IUPAC names for each drug were generated using chemical drawing software Accelrys Draw( 2014 version 4.2. The program allowed insertion of SMILE strings. SMILE strings are line notations describing the structure of chemical compounds using short sequences of letters and numbers. The insertion of these notations into chemical drawing software is useful for rapid structure generation and to derive standardised systematic names like IUPAC names for the molecules drawn.

Three dimensional molecular models were created using Advanced Chemistry Development, Inc. (ACD/Labs@ 2007) 3D-Viewer version 11.01 which also allowed SMILE input. Information was double checked using the online chemical compound database PubChem Compound (NCBI). Rotatable three dimensional representations of the molecules interacting with their cognate receptors were generated from the Protein Data Bank (RCSB PDB: http://www.rcsb.org/pdb/). The protein data bank is a freely accessible large repository of threedimensional biomolecules including proteins and nucleic acids obtained by experimental methods such as x-ray crystallography. The databank is therefore a valuable resource for both pharmacy professionals and students to access biomolecular information and visualise interactions of biomolecules with drug molecules or substrates. The online protein data bank search engine, OCA Browser@ 2013 and Visual Molecular Dynamics VMD C 2013 version 1.9.2 were used to download, manipulate and save three dimensional receptor graphics directly from the online macromolecule archive. These graphical representations were only created for drug molecules that had a relevant PDB entry featuring the drug of interest interacting with the correct receptor for activity in humans at a desirable resolution of $3.5 \AA$ or less. Graphics of the cognate receptors in the ,apo (ee (unbound) and ,holo' (bound) forms were created, as well as a representation of the active site showing the key interactions between the drug and the receptor. The resulting datasheets and visual data of all three chapters were then compiled and saved onto a $\mathrm{CD}$. The $\mathrm{CD}$ also includes a user guide to facilitate future use by educators and students.

\subsection{Questionnaire Validation and Evaluation}

A pre- and post- intervention evaluation analysis was performed on 65 undergraduate Pharmaceutical Science students from all four years of the course. The evaluation tool took the form of a questionnaire. The questionnaire featured five parts that collected data regarding student demographics, student perception, and three sections of medicinal chemistry questions relating to drugs used in infection, endocrine disease and musculoskeletal disorders respectively. The qualitative student perception section featured 7 questions regarding overall attitude towards medicinal chemistry studies, its relevance towards the profession and the use of visual aids in medicinal chemistry lectures. A five point Likert-type scale was assigned for statistical evaluation of pre and post- intervention student perception.

The final three parts of the questionnaire tested the students ${ }^{\text {ee }}$ knowledge of medicinal chemistry concepts within the context of infection, endocrine and musculoskeletal disease. Quantitative data regarding student performance before and after the intervention was collected and evaluated statistically. The questions posed concerned medicinal chemistry concepts and therapeutic issues which are covered in the undergraduate syllabus, students therefore had the background knowledge required to tackle the questions. Questions were also formulated in a manner that both experienced students such as the third and fourth year students and less experienced ones such as the first and second year students could complete, provided the correct reasoning is applied using basic medicinal chemistry principles. The aim was to encourage students to attempt questions and to avoid loss of marks due to questions left unanswered.

The foundations for the questionnaire and intervention lecture design were adapted from the extensive literature review carried out. Questions covered crucial medicinal chemistry definitions, drug modes of action and concepts such as structureactivity relationships, spatial orientation, drug-like properties, stability issues, pharmacophore identification and inhibitory concentrations. Follow up questions then challenged students to apply their knowledge to a clinical scenario in order to provide a clinical context of use to the medicinal chemistry concepts that are inherently abstract, as was carried out in previous studies by Roche, Alsharif and colleagues [12-14]. Some questions featured drug structures depicted in two dimensions typical of traditional didactic lectures. The pre- intervention lecture test acted as a control to assess baseline student performance on subject matter already covered didactically for each year of study. A draft version of the pre- and post- intervention questionnaires were sent to 2 medicinal chemistry educators, 1 pharmaceutical chemistry expert and 2 undergraduate students in order to validate the relevance of the questionnaire to the study. The panel also suggested relevant improvements in questionnaire construction or content where appropriate.

Pre-intervention data was collected by disseminating the pre-intervention questionnaire to 
each year of study and allowing questionnaire completion under supervision to ensure students did not confer answers between themselves and to ensure that internet sources were not accessed. The students were given 45 minutes to complete the questionnaire to their best ability. An intervention lecture was scheduled for each of the four years of undergraduate study. The intervention lecture was carried out a week after the pre-intervention questionnaire. The lecture incorporated both didactic explanations of concepts previously covered in lectures and visual adjuncts in two and three dimensions since background studies show that visual aids in both dimensions are useful as a supplement to traditional lectures and not as an exclusive method of teaching [6-9]. Rotatable two and three dimensional models of the molecules in question were extracted from the database where appropriate as well as three dimensional depictions of the drug interacting with the receptor which allowed a more clear explanation of modes of action and the importance of stereochemistry for drug action. Students were encouraged to participate in the lecture through questions and discussions regarding interpretation of the visuals as an introduction of the concept of „visual literacye [11]. The post-intervention questionnaire was then disseminated and students were given a maximum of 45 minutes, as was allocated for the pre-intervention questionnaire, to complete the task. Statistical analysis was carried out using IBM $®$ SPSS $®$ version 21 @ 92012 . The Friedman and Mann-Whitney statistical tests were applied to qualitative data obtained in part 2 of the questionnaires which dealt with student perception. These tests were carried out in order to statistically conclude whether the use of two and three dimensional visual adjuncts and a more student inclusive teaching method caused a shift in student mentality towards the subject and also towards the use of similar adjuncts in the future.

The Paired Samples t-Test was applied to results obtained from parts 3,4 and 5 of the questionnaires which dealt directly with testing student knowledge regarding subject matter and their ability to rationalise case based problems involving assigned quantitative scores. This test was used to compare mean questionnaire scores before and after the intervention lecture and to assess the impact of the molecular database on student performance, the ability to recall information and application of abstract concepts to clinical scenarios.

\section{Results}

\subsection{Molecular Database}

Completion of phase one of the study lead to the compilation of the datasheets compiled into a user friendly $\mathrm{CD}$ format. The number of molecules included in the study and visual information featured in the database is summarised in Table 1. Datasheets also included drug name, drug class, therapeutic indications, IUPAC name and physicochemical information for each entry. Two and three dimensional models were created for all drug molecules that satisfied inclusion criteria as seen in Figure 1. 3D molecule-receptor graphics were created where a relevant PDB entry conforming to the inclusion criteria specified above could be found. Representations of the receptor in its unbound 'apo" and bound „holo" forms were created. A detailed representation of the active site where the drug docks into the receptor was also created (Figure 1). A user guide was also embedded into the $\mathrm{CD}$ to help prospective users navigate the database and visual software with maximal simplicity.

Table 1. Database contents

\begin{tabular}{ccc}
\hline $\begin{array}{c}\text { BNF } \\
\text { Chapter }\end{array}$ & $\begin{array}{c}\text { No of } \\
\text { Molecules in } \\
\text { the } \\
\text { Datasheet } \\
\text { (including } \\
\text { 2D/3D } \\
\text { models). }\end{array}$ & $\begin{array}{c}\text { No of molecules } \\
\text { with 3D } \\
\text { molecule/receptor } \\
\text { graphics }\end{array}$ \\
\hline Infection & 135 & 58 \\
Musculoskeletal \\
Disorders
\end{tabular}



Figure 1. Example of 2D model, 3D model and 3D graphical representation. Aztreonam monobactam antibacterial in $2 \mathrm{D}$ and $3 \mathrm{D}$, and active site graphic created using Accelrys Draw@ 2014, ACD/Labs@ 2007 3D-Viewer and VMD® 2013 respectively

\subsection{Visual Aid Utility Evaluation}

Part 1 of the questionnaire allowed collection of data regarding student demographics and experience with medicinal chemistry lectures. 65 out of the total 75 undergraduate pharmacy students attended the intervention lecture and completed both pre and post intervention surveys. $23(35 \%)$ of these students were first year students, $12(18 \%)$ were second year students, 19 (29\%) were third year students and 11 $(17 \%)$ were fourth and final year students. Out of the 65 students, only $20(31 \%)$ were male which follows 
the recent trend of the pharmacy course being a predominantly female one. None of the students had a previous scientific degree and all students stated that they had received lectures that covered the concerned topics of infection, musculoskeletal disorders and endocrine disease. First and second year students stated that they had received only elementary instruction regarding these topics. These students were still included in the study as a means of assessing the acquisition of knowledge using these methods.

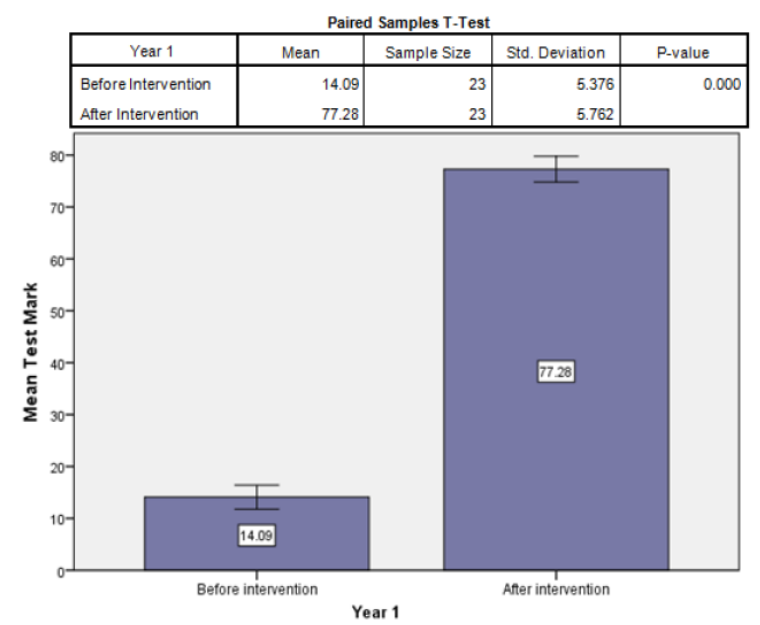

Figure 2. Paired samples t-test results for first year students

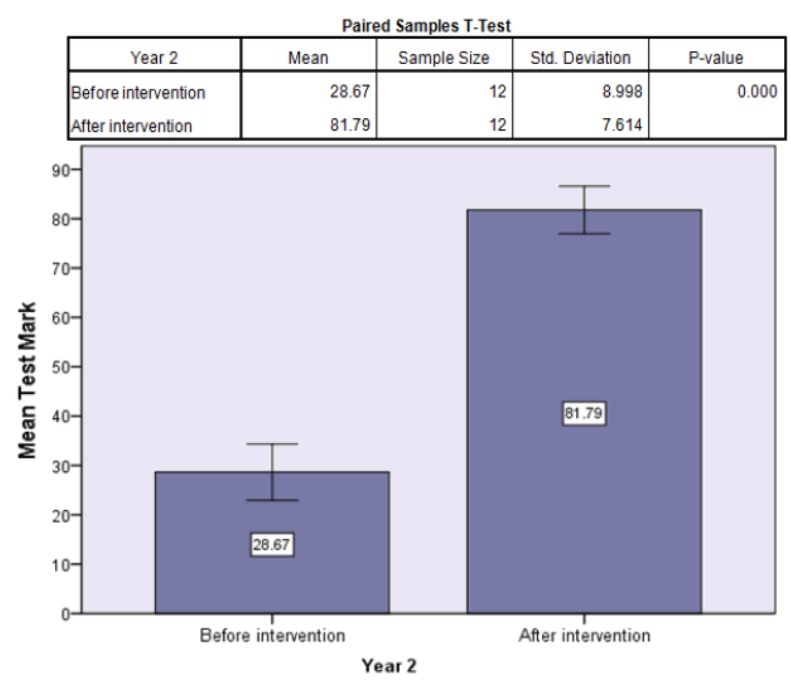

Figure 3. Paired samples t-test results for second year students

In the one-tailed Paired Samples t-Test (Figures 2-5) the mean mark after intervention significantly exceeds the mean mark before intervention for all years of study. In ,year $1^{\text {ee }}$ the mean mark after intervention exceeds the mean mark before intervention by 63.19 points.

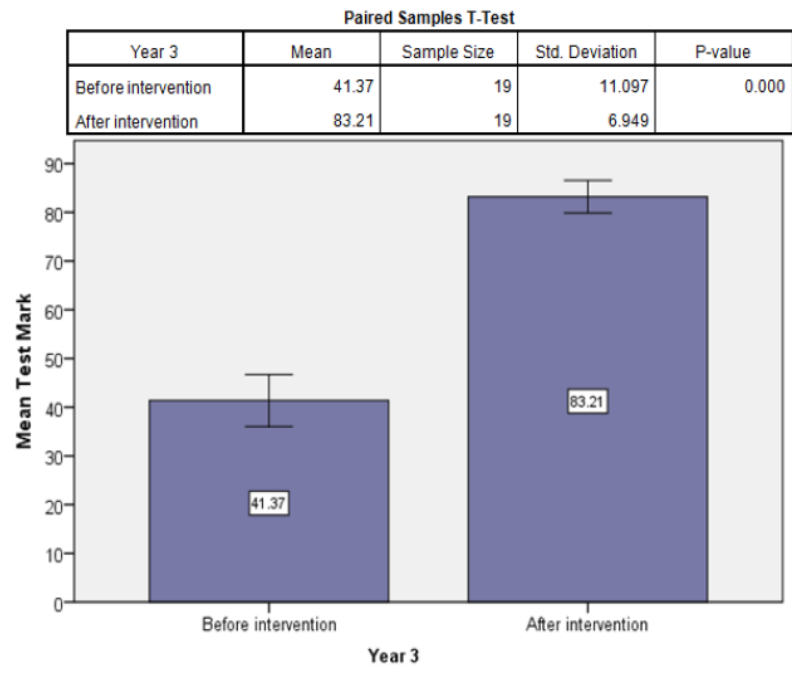

Figure 4. Paired samples T-test results for third year students
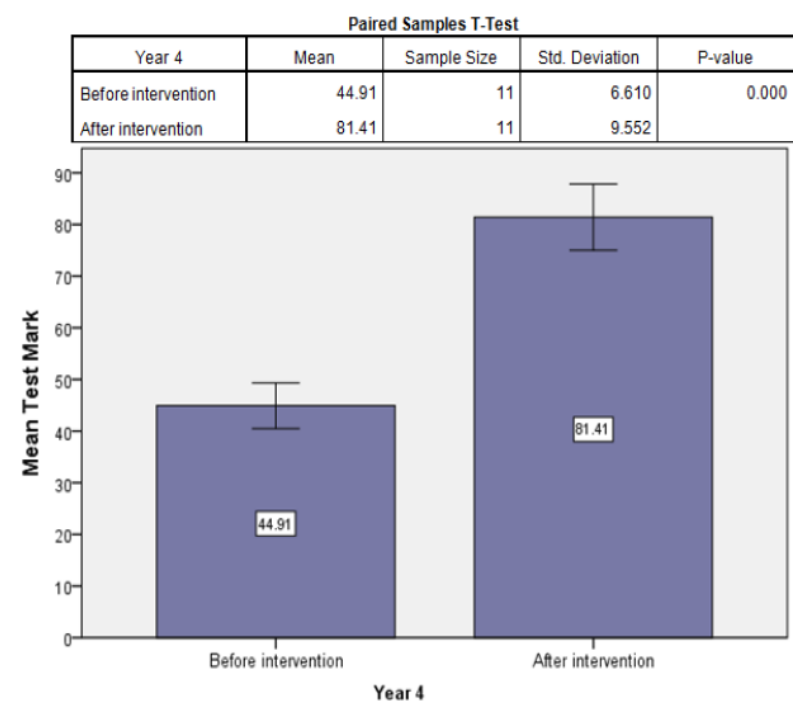

Figure 5. Paired samples T-test results for fourth year students

In ,year $2^{\text {ee }}$ the mean mark after intervention exceeds the mean mark before intervention by 53.12 points. In ,year $3^{\text {ec }}$ the mean mark after intervention exceeds the mean mark before intervention by 41.84 points. In ,year $4^{\text {ee }}$ the mean mark after intervention exceeds the mean mark before intervention by 36.5 points. The improvement in all years of study is statistically significant since the p-values $(<0.001)$ are less than the 0.05 level of significance in all cases. The error bar graph displays the $95 \%$ confidence interval for the actual mean test mark before and after the intervention as can be seen in the figures. The fact that the 2 confidence intervals are disjoint confirms that the improvement was significant and not attributed to chance. 
Table 2. Perception statements

\begin{tabular}{|c|c|}
\hline $\begin{array}{c}\text { Letter } \\
\text { Identifier }\end{array}$ & Statement \\
\hline $\bar{A}$ & $\begin{array}{l}\text { Identifying drug properties based on structure } \\
\text { is important }\end{array}$ \\
\hline B & $\begin{array}{l}\text { Visualizing and deriving conclusions from a } \\
\text { drug's structure is difficult }\end{array}$ \\
\hline C & $\begin{array}{l}\text { Pharmacists should be able to identify } \\
\text { activities and side effects from drug structure }\end{array}$ \\
\hline D & $\begin{array}{l}\text { Concepts of molecular binding, conformation } \\
\text { adopted in vivo and torsion are difficult to } \\
\text { grasp }\end{array}$ \\
\hline $\mathbf{E}$ & $\begin{array}{l}\text { Visualizing and rotating 2D and 3D structures } \\
\text { of a drug is helpful in medicinal chemistry } \\
\text { studies }\end{array}$ \\
\hline $\mathbf{F}$ & $\begin{array}{l}\text { It would be helpful to incorporate } \\
\text { computerised visual aids during medicinal } \\
\text { chemistry lectures }\end{array}$ \\
\hline G & $\begin{array}{l}\text { I would consider using similar visual aids to } \\
\text { aide my personal studies }\end{array}$ \\
\hline
\end{tabular}

Table 3. Friedman test: before intervention

\begin{tabular}{cccc}
\hline Statement & Mean & Standard & P-value \\
& Score & Deviation & \\
\hline A & 3.38 & 0.696 & $<0.001$ \\
B & 2.39 & 0.839 & $<0.001$ \\
C & 2.73 & 0.669 & $<0.001$ \\
D & 2.20 & 0.808 & $<0.001$ \\
E & 3.33 & 0.616 & $<0.001$ \\
F & 3.53 & 0.638 & $<0.001$ \\
G & 3.23 & 0.760 & $<0.001$ \\
\hline
\end{tabular}

Table 4. Friedman test: after intervention

\begin{tabular}{cccc}
\hline Statement & Mean & $\begin{array}{c}\text { Standard } \\
\text { Deviation }\end{array}$ & P-value \\
& Score & 0.571 & $<0.001$ \\
\hline $\mathbf{A}$ & 3.63 & 1.268 & $<0.001$ \\
$\mathbf{B}$ & 1.72 & 0.683 & $<0.001$ \\
$\mathbf{C}$ & 3.26 & 1.002 & $<0.001$ \\
D & 2.34 & 0.477 & $<0.001$ \\
E & 3.74 & 0.469 & $<0.001$ \\
F & 3.75 & 0.641 & $<0.001$ \\
G & 3.65 & & \\
\hline
\end{tabular}

The Friedman and Mann-Whitney tests were applied to data emanating from part 2 of the questionnaire which dealt with student perception.
The statements posed to students to gather changes in student perception between pre- and postintervention assessments can be found in Table 2. In the Friedman test results (see Tables 3 and 4) we can see that the mean rating scores for both before and after intervention questionnaires vary significantly since the p-value $(<0.001)$ is less than the 0.05 level of significance. In the Mann-Whitney test (see Table 5 and Figure 6) the null hypothesis specifies that there is no change in the mean rating score after intervention and this is accepted if the p-value exceeds the 0.05 level of significance. The alternative hypothesis specifies that the mean rating score before and after intervention varies significantly. This is accepted if the p-value is less than the 0.05 level of significance. P-values were below 0.05 for all statements except statement , $\mathrm{D}^{\text {ee }}$. Therefore all statements bar „D $\mathrm{D}^{\text {ee }}$ showed significantly different rating scores when comparing pre and post intervention perception scores.

Table 5. Mann-Whitney test results

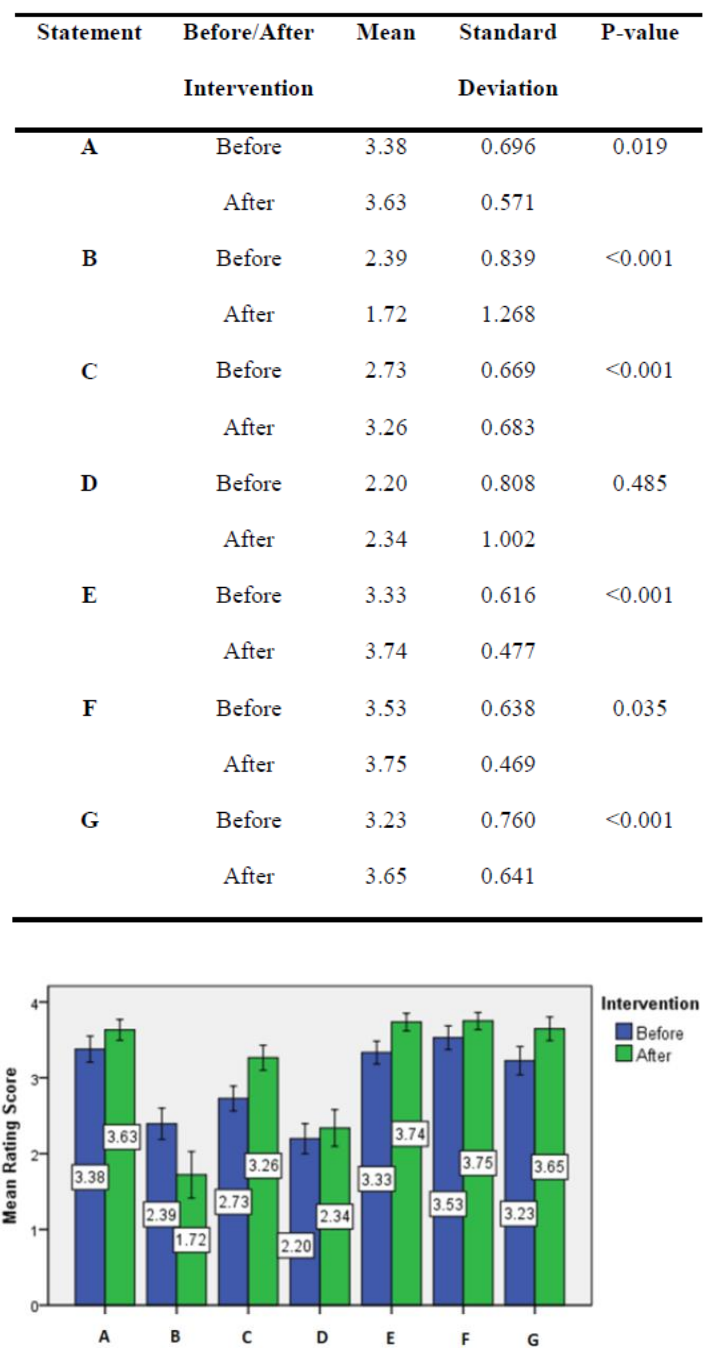

Figure 6. Mann-Whitney Test Results 


\section{Discussion}

The purpose of this study was to evaluate the impact of the inclusion of two and three dimensional visual adjuncts to traditional medicinal chemistry lecturing methods within the undergraduate pharmacy syllabus. This study is unique when compared to similar previous studies in the field due to the fact that it combines successful strategies from established studies. Through a combination of these strategies, the study aimed at providing a holistic and realistic representation of the effects of the careful and intelligent use of visual adjuncts as an aid to the didactic approach among pharmacy students. Overall, the study showed concordant results to those obtained in previous studies.

In the one-tailed Paired Samples t-Test (Figures 2-5) the null hypothesis specifies that there is no change in the mean test mark after intervention and this is accepted if the p-value exceeds the 0.05 level of significance. The alternative hypothesis specifies that the mean mark improves significantly after intervention and is accepted if the p-value is less than the 0.05 level of significance. The $p$-values $(<0.001)$ are less than the 0.05 level of significance for all years of study. The alternative hypothesis was accepted and therefore students showed improved performance and understanding of the subject matter. Students in 'year 1' of study obtained the lowest mean mark before intervention closely followed by students in 'year 2'. As expected these students showed the largest improvement in mean test scores following the intervention since they had a larger margin for improvement. For students in the first two years of study, the medicinal chemistry concepts tackled in the pre-intervention questionnaire were relatively novel and students therefore found it difficult to apply these concepts to the case study questions provided. During the lecture the students showed in depth understanding of the basic concept and participated in discussions during projection of 2D and 3D visuals and explanations. As seen from the t-test result, when challenged with the same questions post-intervention lecture, student performance improved significantly. It can be implied that students gained a greater depth of comprehension for the subject matter and felt confident answering several questions that were left blank in the pre-intervention questionnaire. An improvement in student performance was identified. Students in 'year 3' showed the highest postintervention questionnaire average score, closely followed by 'year 4' students. 'Year 4' students showed the smallest difference in mean scores before and after the intervention, and also showed the highest pre-intervention average test score. This can be interpreted based on the fact that in third year, the medicinal chemistry syllabus emphasises heavily on principles included in the study questionnaire and therefore post-intervention these students were able to recollect information which was recently obtained through didactic methods with the help of visualisation methods.

Fourth year students showed the highest amount of baseline knowledge of the subject matter, which was expected due to their longer exposure to medicinal chemistry lectures. Although third and fourth year students showed superior baseline knowledge, significant improvement of average test scores was still obtained through exposure to visual aids during the intervention lecture. Through the use of 2D/3D visualisation of molecular examples emphasising medicinal chemistry concepts along with traditional didactic explanation, more experienced students were able to recall and reason out case based questions more successfully postintervention due to enhanced understanding and recollection provided by visual adjuncts to traditional teaching to a larger degree than prior to the intervention. Less experienced students showed the largest improvement due to an enhanced understanding of abstract medicinal chemistry concepts that were explained in a tangible, visual manner using the aids.

The Friedman test was used to compare the mean rating scores allocated to the set of statements used in the student perception section of the questionnaire (part 2). A Likert-type scale was applied to the statements where the mean rating scores ranged from 0 to 4. ' 0 'corresponds to 'strongly disagree', ' 1 'corresponds to 'disagree' '2'corresponds to 'neutral', ' 3 'corresponds to 'agree' and '4'corresponds to 'strongly agree'. The null hypothesis specifies that the mean rating scores provided to statements are similar and is accepted if the p-value exceeds the 0.05 level of significance. The alternative hypothesis specifies that the mean rating scores provided to statements vary significantly and is accepted if the p-value is less than the 0.05 level of significance. In the Friedman test results corresponding to the 'before intervention' sample (see Table 3 ), the mean rating score provided to statement ' $\mathrm{f}$ ': It would be helpful to incorporate computerised visual aids during medicinal chemistry lectures is the largest, indicating highest agreement. This is followed by statement ' $a$ ': Identifying a druges properties based on its structural formula is important, 'e': Visualising and rotating the 2D and 3D structures of a drug is helpful in medicinal chemistry studies, 'g': I would consider using similar computational visual aids of molecular structure of drugs and their receptors to aid my personal studies, 'c': Pharmacists should be able to identify certain activities or side effects of a drug according to its structure , 'b': Visualising and deriving conclusions from a drug's three-dimensional structure is difficult and ' $d$ ': Concepts of molecular binding, conformations adopted in vivo and torsion are 
difficult to grasp. These mean rating scores vary significantly since the p-value $(<0.001)$ is less than the 0.05 level of significance. In the Friedman test results corresponding to the 'after intervention' sample (see Table 4), the mean rating score provided to statement ' $\mathrm{f}$ ': It would be helpful to incorporate computerised visual aids during medicinal chemistry lectures is once again the largest, with a higher rating score than before intervention, indicating higher agreement. This is followed by statement 'e', 'g', 'a', 'c', 'd' and 'b'. These mean rating scores vary significantly since the p-value $(<0.001)$ is less than the 0.05 level of significance. The Mann-Whitney test (see Table 5 and Figure 6) is used to compare mean rating scores provided to each statement before and after the intervention. The null hypothesis specifies that there is no change in the mean rating score after intervention and this is accepted if the $p$ value exceeds the 0.05 level of significance. The alternative hypothesis specifies that the mean rating score before and after intervention varies significantly. This is accepted if the p-value is less than the 0.05 level of significance. There was a statistically significant reduction in mean rating score for statement ' $b$ ': Visualising and deriving conclusions from a drug's structure is difficult, signifying decreased agreement post intervention. This statement showed the largest difference between pre and post mean rating scores with a difference of -0.67 . This leads to the interpretation that the intervention reduced the perceived level of difficulty associated with visualisation of molecules and interpreting 2D and 3D models. This shows improved confidence in interpreting drug structure and visual literacy which are important pillars in improvement of mentality towards the subject matter. There was a significant increase in mean rating score for statements, 'a', 'c', 'e', 'f' and ' $g$ ' after intervention. All these statements saw increased agreement post intervention. Statement 'c' showed the largest increase in agreement between pre and post average scores $(0.53)$, followed by statement ' $\mathrm{g}$ ' (0.42). This suggests a shift in student mentality regarding the importance of medicinal chemistry to the pharmaceutical profession and use of visual aids in order to facilitate medicinal chemistry studies. The increment in mean rating score for statement 'd': Concepts of molecular binding, conformation adopted in vivo and torsion are difficult to grasp was not significant since the p-value exceeds the 0.05 level of significance. This is clearly displayed by the errors bars shown (Figure 6.) where confidence level brackets overlap each other which is a sign of lack of significance. A possible reason for this could be unclear wording of the question without clarification during the intervention.

Drugs with peptide structures were not included in the molecular database. The main problems encountered with such drugs were the inability of these drug molecules to be supported and manipulated by the chemistry software due to the considerable number of atoms present and the inability of chemical drawing software to correctly show all bond angles present. Combination therapy entries were also omitted due to complications with regards to deriving relevant information for the drug pair, however, where possible, the individual entities were allocated their own drug entry. The PDB does not currently contain PDB depositions for all drugs in contemporary use. In fact, it was not possible to generate three dimensional graphical representations of ligand-protein interactions for all molecules included in the datasheets. The detailed threedimensional structures of proteins and nucleic acids are determined mainly through X-ray crystallography which obtains high resolution depositions, however nuclear magnetic resonance and cryo-electron microscopy also account for a smaller percentage of structures. The crystallography method is the preferred method to generate PDB data, however it is a highly complex and time consuming process where crystal formation and stability limits its application. Furthermore, PDB entries included required an appropriate deposition that fulfilled the following inclusion criteria. Firstly, the drug molecule in the deposition must interact with the correct cognate receptor responsible for clinical activity within human, and secondly, the deposition must be at a reasonable resolution $(<3.5 \AA$ ) which further excluded some PDB entries due to undesirable image quality. The tool was initially intended to be available in an online format; however major problems were encountered when attempts at uploading the large file sizes were made. Security restrictions in recent uploading software updates denied access of uploaded molecules by third party computer devices. The most convenient vehicle for presentation of the databases and for the purpose of the study was therefore a multimedia CD.

\section{Conclusion}

Overall, the incorporation of the two and three dimensional molecular database as a visual aid in the medicinal chemistry intervention lecture showed a statistically significant improvement in student performance in all years of study. Furthermore, through both statistical analysis of perception related questions in the questionnaire and direct feedback after the lecture, students showed an improvement in enthusiasm towards the subject and expressed that the learning experience was more pleasant due to clear concept delivery and direct student involvement encouraged by the visual aids. Students stated that they would seriously consider using visual adjuncts in personal studies if made available. 


\section{References}

[1] F. Khan, M. J. Deimling, A. Philip, Medicinal Chemistry and the Pharmacy Curriculum. American Journal of Pharmaceutical Education. 2011, 75(8), pp. 161.

[2] C. Wu, J. Foos. Making chemistry fun to learn. Literacy Information and Computer Education Journal. 2010 March, 1(1), pp. 3-7

[3] R. E. Wileman. (1993) Visual communicating. Englewood Cliffs, Educational Technology Publications, New Jersey.

[4] I. Carvalho, A. Borges, L. Bernardes, Medicinal Chemistry and Molecular Modeling: An Integration for the Teaching of Drug Structure-Activity Relationship and the Molecular Basis of Drug Action. Journal of Chemical Education. 2005, 82 (4) , pp. 588-596.

[5] M. Abraham, V. Varghese, H. Tang. Using Molecular Representations to Aid Student Understanding of Stereochemical Concepts. Journal of Chemical Education. 2010, 87(12), pp. 1425-1429.

[6] P. Zhou, Z. Shang. 2D Molecular Graphics: a Flattened World of Chemistry and Biology. Briefings in Bioinformatics. 2009, 10(3), pp. 247-258.

[7] J. S. Krajcik, E. Soloway, H. K. Wu. Promoting Conceptual Understanding of Chemical Representations: Students"e Use of a Visualization Tool in the Classroom. Journal of Research in Science Teaching. 2000, 38, pp. 821-842.

[8] M. J. Ingram, A. J. Long, G. P. Moss, L. A. Sagoe, M. H. Sosabowski. Pharmacy Student Perception of Educational Media Tools. Pharmacy Education. 2007, 7(1), pp. 77-82.

[9] T. I. Poirier. A Seminar Course on Contemporary Pharmacy Issues. American Journal of Pharmaceutical Education. 2008, 72 (2), pp. 30.

[10] K. L. Vavra, V. Janjic-Watrich, K. Loerke, L. M. Phillips, S. P. Norris, J. Macnab. Visualization in Science Education. Alberta Science Education Journal 2011 January, 41(1), pp. 22-30.

[11] S. Stokes. Visual Literacy in Teaching and Learning: A literature Perspective. Electronic Journal for the Integration of Technology in Education. 2003, 1(1), pp.1019.

[12] V. F. Roche, M. J. Aitken, S. W. Zito. Evaluation of Computerized Medicinal Chemistry Case Study Modules as Tools to Enhance Student Learning and Clinical Problem-Solving Skills. American Journal of Pharmaceutical Education. 1999, 63(3), pp. 289-295.

[13] N. Z. Alsharif, R. Chapman, K. A. Galt, A. Mehanna, A. M Ogunbadeniyi. Instructional Model to Teach Clinically Relevant Medicinal Chemistry. American Journal of Pharmaceutical Education. 2006, 70(4): 91.
[14] N. Z. Alsharif, K. A. Galt. Evaluation of an Instructional Model to Teach Clinically Relevant Medicinal Chemistry. American Journal of Pharmaceutical Education. 2008 Apr; 72 (2): 31.

[15] Joint Formulary Committee. (2017) British National Formulary 72. BMJ Group and Pharmaceutical Press, London. 